\title{
Vertebral Compression Fractures as a Presenting Feature of AcuteLymphoblastic Leukemia
}

\author{
APARNA DAS, ${ }^{1}$ FARNAZ NOBI, ${ }^{2}$ GOBINDA BANIK, ${ }^{3}$ MD. AZIZUL KAHHAR ${ }^{4}$
}

\begin{abstract}
Acute lymphoblastic leukemia is one of the most common malignancies in children and adolescent accounting for $30 \%$ of all the cancers. We report an interesting case of Acute lymphoblastic leukaemia(ALL) with an unusual presentation. This 15-year-old girl came with a progressive low back pain for three months following a history of fall for 5 month ago with intact consciousness. The physical examination showed gibbus over lumbar spine with tenderness. Images showed generalized osteopenia with multiple vertebral body collapse. Complete blood count revealed pancytopenia with raised ESR without any blast cell. Bone marrow aspirate was suggestive of ALL. Young ALL patients usually present with symptoms due to cytopenias, fever and bone pains. Although asymptomatic skeletal involvement may be present in patients with ALL, rarely patients present with pathological fractures. Therefore a high index of suspicion is needed to diagnose such case.
\end{abstract}

Keywords: Vertebral collapse, Pancytopenia, Acute lymphoblastic leukemia

\section{Introduction}

Bone and joint pain may be presenting symptoms in $25 \%$ patients in acute leukemia ${ }^{1}$ whereasgeneralized osteopeniaand vertebral complications are less common ${ }^{2}$. Children usually present with symptoms due to cytopenias,organomegaly, lymphadenopathy and bone pains.Children and adolescent with leukemia may initially present to the rheumatologist with leukemic arthritis. ${ }^{3}$ Whereas generalised osteopenia and vertebral complications are less common. ${ }^{4,5}$ Skeletal manifestations presenting in the form of bone pains, refusal to walk etc. may be seen in $40-60 \%$ of patients. However there are very few reports of childhood ALL presenting with severe osteopenia.We report an interesting case of a 15 year old girl who presented with severe low back pain and vertebral compression.

\section{Case summary}

A 15- year- old girl presented with progressive noninflammatory low back pain for 3 months without any improvement with analgesic following a history of fall from chair 5 month ago. For the last two months pain was so severe

1. Assoc. Prof., Dept. of Medicine, Dhaka Medical College.

2. Post graduate trainee, Dept. of Medicine, Dhaka Medical College.

3. Asst. Prof., Dept. of Medicine, Dhaka Medical College.

4. Professor, Dept. of Medicine, Dhaka Medical College.

Corresponding author: Dr.. Aparna Das, Assoc. Prof., Dept. of Medicine, Dhaka Medical College. Email: aparna_0191@, yahoo.com. that patient refused to move orwalk. She also had intermittent fever, generalized weakness and weight loss for last 2 months. Her motor and mental development was normal for age. There was no significant past and family history. Clinical examination revealed pale, afebrile, emaciated, presence of lumbar spinal gibbus with tenderness without lymphadenopathy or hepatomegaly and splenomegaly.

The radiography of the spine observed diffuse vertebral body collapse with osteopenia.

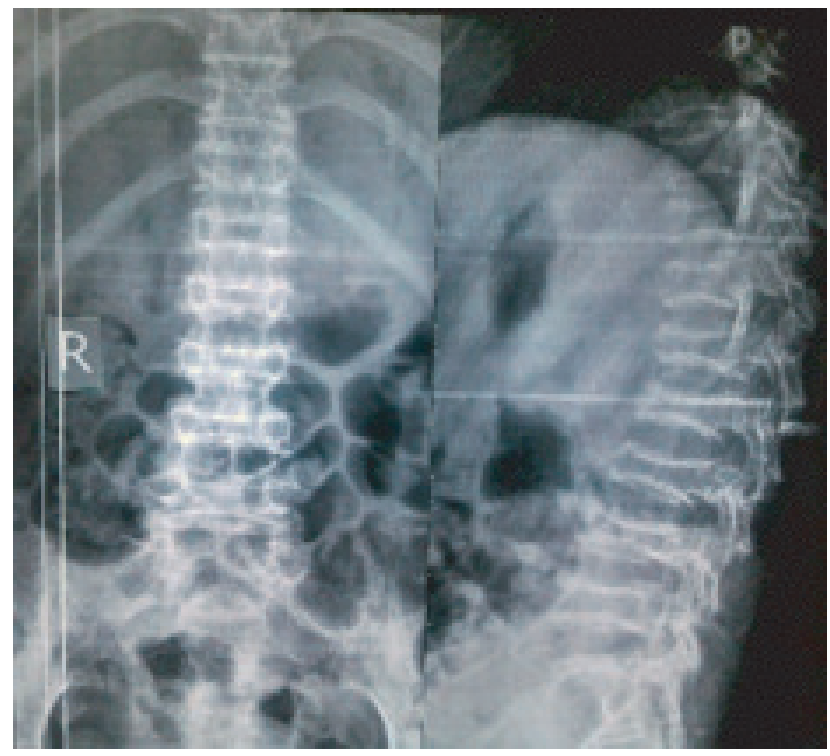

Fig.-1: Generalized osteopenia of spine with multiple vertebral compressions. 
The blood count showed: Hemoglobin $9.2 \mathrm{gm} / \mathrm{dl}$, Leukocyte $1.50 \times 10^{9} / \mathrm{L}$, Neutrophils $13 \%$,

Lymphocyte $84 \%$, Monocyte $02 \%$, Eosinophils $01 \%$ without any blasts, Platelet count $20 \times 10^{9} / \mathrm{L}$ and ESR $55 \mathrm{~mm}$ in $1^{\text {st }}$ hour. PBF revealed pancytopenia. Bonemarrow aspirate showed Acute Lymphoblastic Leukemia (ALL-L2).

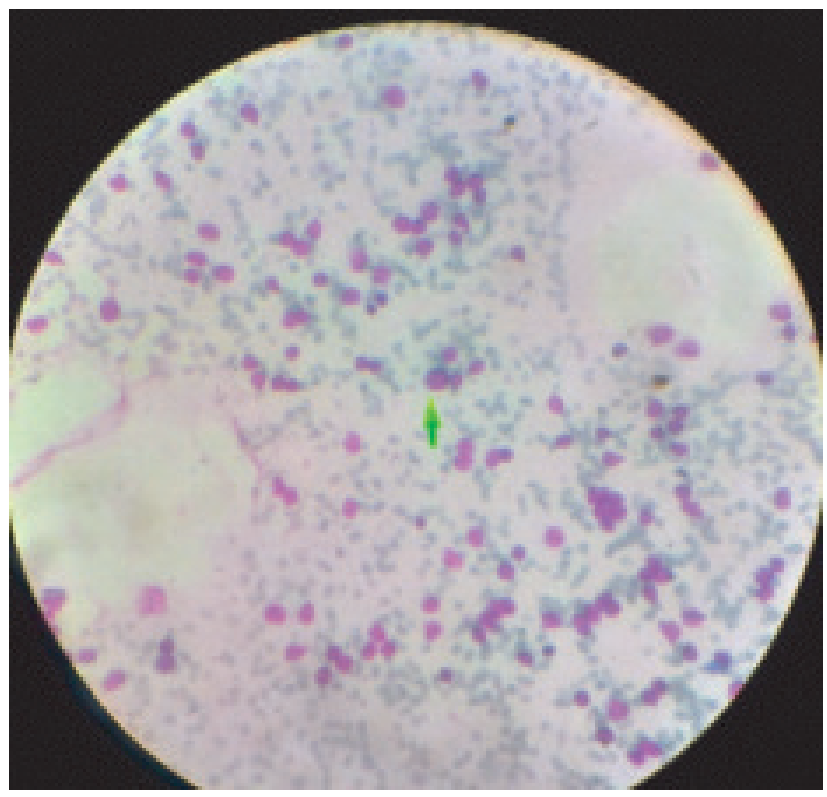

Fig.-2: Bone marrow smear showing numerous Lymphoblasts(arrow).

Chest x-ray normal, Serum calcium level $9.5 \mathrm{mg} / \mathrm{dl}$ (Ref. range 8.4-10.2 mg/dl), Serum albumin $38.9 \mathrm{gm} / \mathrm{L}$, Vitamin D $27.1 \mathrm{sg} / \mathrm{ml}$ (Ref-8.8-46.3 sg/ml),PTH $3.67 \mathrm{pg} / \mathrm{ml}(7-53$ $\mathrm{pg} / \mathrm{ml}$ ). BMD showed: Age matched Z score -3.8. USG of whole abdomen revealed: Splenomegaly with prominent intraabdominal lymphnodes.

\section{Discussion}

Common manifestations of childhood ALL are fever,bony pains and symptoms due to cytopenias. Although asymptomatic skeletal involvement may be present in patients with ALL, rarely patients present with pathological fractures. Therefore a high index of suspicion is needed to diagnose such case. The osteoarticular manifestations which are frequent in the ALL are osteopenia, lysis of bone, lesions of osteosclerosis and periosteal reactions have been described. ${ }^{3}$ Vertebral compression is rarely afirst manifestation of ALL.

Radiological evidence of demineralization during course of leukemia can be caused by disease, inactivity, steroids or other antileukemic drugs or to abnormalities in mineral homeostasis. ${ }^{4}$ Spinal involvement may be a presenting feature despite normal peripheral blood counts. ${ }^{5,6}$ In our case first symptoms was non-inflamatory low back pain without having features of cytopenias.Imagings revealed generalized osteopenia of spine with multiple vertebral compressions. So, we considered the differential diagnoses of multiple vertebral compressions like, hyperparathyroidism, tuberculosis, secondaries and haematological malignancies. But, blood reports revealed that this girl having cytopenias with high ESR and PBF showed only pancytopenia without blast cells. Bone marrow confirmed diagnosis ALL. ALL who present with leucopenia, severe constant pain and a long interval between the onset of symptoms and diagnosis carries an unfavorable outlook. Heinrich et al suggested that duration of symptoms prior to diagnosis influences outcome rather than the nature of skeletal radiographic abnormalities. ${ }^{7}$ Antileukemic treatment usually results in rapid symptomatic relief as well as radiographic evidence of bony remodeling.

\section{Conclusion}

We wish to highlight that vertebral compressions are very rare presenting feature of a patient with pancytopenia having ALL. Physicians need to keep a high index of suspicion to suspect ALL in such patients, particularly if there is worsening back pain with associated with vertebral compression with pancytopenia.

\section{Conflict of interest: None}

\section{References}

1. Vassilopoulou-Sellin R, Ramirez I. Severe osteopenia and vertebral compression fractures after complete remission in an adolescent with acute leukemia. Am J Hematol. 1992;39:142-143.

2. Cohn SL, Morgan ER, Mallette LE. The spectrum of metabolic bone disease in lymphoblastic leukemia.Cancer. 1987;59:346-350.

3. Amaresh Reddy P, Alok S, Suresh V .Osteoporosis and Vertebral Compression Fractures as a Presenting Feature Of Childhood Acute Lymphoblastic Leukemia. J Endocrinol Diabetes Obes. 2014;2(2):1021.

4. Pandya NA, Meller ST, MacVicar D et al. "Vertebral Compression Fractures in Acute Lymphoblastic Leukaemia and Remodeling after Treatment," Archives of Disease in Children, 2001;85(6):492-493.

5. Santangelo JR \& Thomson JD" Childhood Leukemia Presenting with Back Pain and Vertebral Compression Fractures," The American Journal of Orthopedics, 1999; 28(4):257-260.

6. Cherkaoui S, Hmimech A, Madani A and Benchekroun A." Vertebral Body Collapse at the Onset of Acute Lymphoblastic Leukemia," Pediatrics Research International Journal. 2014 Article ID 955855, DOI:10.5171/2014.955855

7. Heinrich SD, Gallager, D'Warrior R.The prognostic significance of the skeletal manifestations of ALL of childhood. J Pediatr Orthop.1994;14:105-111. 